\title{
(6) OPEN ACCESS \\ Identifying clusters of falls-related hospital admissions to inform population targets for prioritising falls prevention programmes
}

\author{
Caroline F Finch, ${ }^{1}$ Karen Stephan, ${ }^{2}$ Anna Wong Shee, ${ }^{1}$ Keith Hill, ${ }^{3}$ Terry P Haines, ${ }^{4}$ \\ Lindy Clemson, ${ }^{5}$ Lesley Day ${ }^{2}$
}

${ }^{1}$ Australian Centre for Research into Injury In Sport and its Prevention (ACRISP), Federation University Australia, Ballarat, Victoria, Australia

${ }^{2}$ Monash Injury Research Institute (MIRI), Monash University, Clayton, Victoria, Australia

${ }^{3}$ Faculty of Health Sciences, School of Physiotherapy and Exercise Science,

Curtin University, Perth, Western Australia, Australia ${ }^{4}$ Allied Health Research Unit, Monash Health, Monash University, Clayton, Victoria, Australia

${ }^{5}$ Ageing, Work \& Health Research Unit, Faculty of Health Sciences, The University of Sydney, Sydney, New South Wales, Australia

\section{Correspondence to}

Professor Caroline Finch, Australian Centre for Research into Injury In Sport and its Prevention (ACRISP), Federation University Australia, PO Box 663, Ballarat, Victoria, Australia;

c.finch@federation.edu.au

Received 25 June 2014 Revised 10 December 2014 Accepted 7 January 2015 Published Online First 24 January 2015

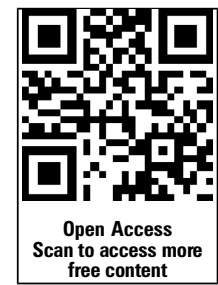

CrossMark

To cite: Finch CF, Stephan K, Shee AW, et al. Inj Prev 2015;21:254-259.

\section{ABSTRACT}

Background There has been limited research investigating the relationship between injurious falls and hospital resource use. The aims of this study were to identify clusters of community-dwelling older people in the general population who are at increased risk of being admitted to hospital following a fall and how those clusters differed in their use of hospital resources. Methods Analysis of routinely collected hospital admissions data relating to 45374 fall-related admissions in Victorian community-dwelling older adults aged $\geq 65$ years that occurred during 2008/2009 to 2010/2011. Fall-related admission episodes were identified based on being admitted from a private residence to hospital with a principal diagnosis of injury (International Classification of Diseases (ICD)-10-AM codes S00 to T75) and having a first external cause of a fall (ICD-10-AM codes W00 to W19). A cluster analysis was performed to identify homogeneous groups using demographic details of patients and information on the presence of comorbidities. Hospital length of stay (LOS) was compared across clusters using competing risks regression.

Results Clusters based on area of residence, demographic factors (age, gender, marital status, country of birth) and the presence of comorbidities were identified. Clusters representing hospitalised fallers with comorbidities were associated with longer LOS compared with other cluster groups. Clusters delineated by demographic factors were also associated with increased LOS.

Conclusions All patients with comorbidity, and older women without comorbidities, stay in hospital longer following a fall and hence consume a disproportionate share of hospital resources. These findings have important implications for the targeting of falls prevention interventions for community-dwelling older people.

\section{INTRODUCTION}

Falls among older adults are an increasing problem, with up to $30 \%$ of older people living in the community falling each year ${ }^{1}$ and around 1 in 10 of all falls resulting in fracture. ${ }^{1}{ }^{2}$ Falls can have severe consequences, resulting in losses in confidence, mobility and independence contributing to decreased quality of $\operatorname{life}^{3}{ }^{4}$ and they can also have significant financial implications. For example, the predicted fall-related healthcare burden in Australia is expected to increase to at least $\mathrm{A} \$ 1375$ million per year over the next few decades unless effective prevention and lower treatment costs occur. ${ }^{56}$
Despite substantial evidence for effective falls prevention interventions for community-dwelling older people, ${ }^{1}$ both numbers and rates of all falls-related hospital admissions among older people have been increasing in developed countries. $^{7}$ This suggests that the research evidence has not yet been incorporated into falls prevention practice and the public health benefit of falls prevention is yet to be fully realised. Previous research has shown a 'one-size-fits-all' approach to falls prevention is not the solution and that falls prevention interventions may need to be specific to particular groups to be effective. ${ }^{8}$ A key challenge that now exists for falls prevention in older people is the effective delivery and uptake of evidence-based falls prevention interventions. ${ }^{9} 10$

There has been considerable research on identifying people who have an increased risk of falls and fall-related injury. In contrast, there has been limited research investigating the relationship between injurious falls and health outcomes. For example, some groups of people at high falls risk may have longer hospital stays following a fall event, and hence use more hospital resources and incur greater healthcare costs. Understanding the factors that impact on the healthcare outcomes associated with injurious falls, such as comorbidity, could be used to optimise the targeting and delivery of falls prevention interventions, assuming that falls prevention interventions are equally beneficial across these subpopulations. ${ }^{8} 11$

The aims of this study were to identify clusters of community-dwelling older people in the general population who are at increased risk of being admitted to hospital following a fall and identify how those clusters differed in their use of hospital resources, based on hospital length of stay (LOS). Identification of these clusters can inform the targeting of future well-defined population-level interventions and programmes for falls prevention to reduce the impact of fall-related injuries on hospital resources. The contribution of the presence of comorbidities in individuals within these clusters was of particular interest. This study is part of a larger partnership project aimed at generating and integrating evidence to facilitate better targeting of available falls prevention investment. ${ }^{12}$ Other evidence generated from that partnership project will be used to facilitate improved targeting of evidence-based falls interventions to groups of older people who are most frequently hospitalised for fall-related conditions. 


\section{METHODS}

\section{Data source and case selection criteria}

The Victorian Admitted Episodes Data Set (VAED) was used for this research. The VAED comprises data on episodes of patient admission to acute hospitals, both public and private, in the state of Victoria, Australia. Victoria is the second most populous state in Australia with a population of 5.713 million. ${ }^{13}$ A large proportion of the Victorian population (73\%) live in the metropolitan capital city, Melbourne. ${ }^{13}$ Episodes of admissions for falls in Victorian community-dwelling adults, aged $65+$ years, that occurred during the three financial years 2008/2009 through 2010/2011 were identified on the basis of being admitted from a private residence to hospital with a principal diagnosis of injury (International Classification of Diseases (ICD)-10-AM codes S00 to T75) and having a first external cause of a fall (ICD-10-AM codes W00 to W19). Episodes were excluded if the fall occurred in an aged care facility, if the patient was indigenous or if they resided outside of Victoria. The effect of comorbidity begins at a younger age in indigenous people, so they were excluded in this analysis of $\geq 65$ year olds. Fewer than $0.3 \%$ of people aged $65+$ years are indigenous. ${ }^{13}$ Duplicate records and readmissions from a previous falls-related incident were reduced by excluding admissions to a rehabilitation hospital, transfers from other hospitals, readmissions to the same hospital within 30 days and admissions that were coded as statistical separations.

Demographic and clinical information were extracted from the VAED for each episode of care. LOS was measured from the time of admission to separation, for the first admission for a fallrelated injury. The prevalence of comorbidity was based on the presence of at least one recorded comorbidity at the time of the

Table 1 Patient characteristics corresponding to episodes of admissions for falls in community-dwelling older adults

\begin{tabular}{|c|c|c|c|}
\hline & $\begin{array}{l}\text { Melbourne } \\
\text { metropolitan area } \\
\text { N (\%) }\end{array}$ & $\begin{array}{l}\text { Regional/rural } \\
\text { Victoria } \\
\mathrm{N}(\%)\end{array}$ & $\begin{array}{l}\text { Total } \\
\text { N (\%) }\end{array}$ \\
\hline \multicolumn{4}{|l|}{ Sex } \\
\hline Male & 10297 (31.2) & 3866 (31.4) & 14163 (31.2) \\
\hline Female & $22757(68.8)$ & 8454 (68.6) & 31211 (68.8) \\
\hline \multicolumn{4}{|l|}{ Age group (years) } \\
\hline $65-74$ & $7947(24.0)$ & $2958(24.0)$ & $10905(24.0)$ \\
\hline $75-84$ & $13472(40.8)$ & $5217(42.3)$ & $18689(41.2)$ \\
\hline $85+$ & $11635(35.2)$ & 4145 (33.6) & 15780 (34.8) \\
\hline \multicolumn{4}{|l|}{ Marital status } \\
\hline $\begin{array}{l}\text { Never married, } \\
\text { widowed, } \\
\text { divorced, } \\
\text { separated }\end{array}$ & $17393(52.6)$ & $6605(53.6)$ & 23998 (52.9) \\
\hline $\begin{array}{l}\text { Currently married, } \\
\text { de facto }\end{array}$ & $14886(45.0)$ & $5437(44.1)$ & $20323(44.8)$ \\
\hline Missing & 775 (2.3) & $278(2.3)$ & $1053(2.3)$ \\
\hline \multicolumn{4}{|l|}{ Country of birth } \\
\hline $\begin{array}{l}\text { Australia } \\
\text { (including external } \\
\text { territories) }\end{array}$ & 19124 (57.9) & $9998(81.2)$ & $29122(64.2)$ \\
\hline All other countries & $13240(40.1)$ & $2226(18.1)$ & 15466 (34.1) \\
\hline Missing & $690(2.1)$ & $96(0.8)$ & $786(1.7)$ \\
\hline \multicolumn{4}{|l|}{ Comorbidities } \\
\hline None & $24457(74.0)$ & 9096 (73.8) & 33553 (73.9) \\
\hline One or more & $8597(26.0)$ & $3224(26.2)$ & $11821(26.1)$ \\
\hline Total & 33054 (72.8) & $12320(27.2)$ & $45374(100.0)$ \\
\hline
\end{tabular}

patient's first fall-related injury admission. Comorbidities were defined as medical conditions present at the time of admission and identified as a primary or pre-existing condition, or as an associated condition not treated during the stay in hospital. Medical conditions defined as comorbidities in this study were those included in the Charlson comorbidity index (CCI). ${ }^{14}$ In addition, we included conditions identified as falls risk factors in the literature for which ICD-10 AM coding was available.

\section{Statistical analysis}

A cluster analysis was performed to identify homogeneous groups using demographic details of patients as well as information on comorbidities. A two-step cluster analysis procedure with a log-likelihood distance measure ${ }^{15} 16$ within IBM SPSS Statistics (V.20) was used because it is appropriate for large data sets with categorical data. The number of clusters was determined automatically using the Bayesian information criterion. ${ }^{16}$ The average silhouette measure of cohesion and separation (which ranges from -1 to +1 ) was used to indicate overall goodness of fit. Positive values indicate that the average distance between cases in a cluster is smaller than the average distance to cases in other clusters, and are thus desirable. ${ }^{17}$ There is little guidance in the published literature regarding interpretation of the magnitude of the average silhouette. That which is available is generally based on the experience of researchers in their own particular field. A generally accepted criterion is that if the silhouette measure is $<0.2$, then the quality of the average silhouette measure across the whole sample is considered poor, between 0.2 and 0.5 indicates a fair solution and $>0.5$ is a good solution. ${ }^{18}$

Initial attempts to identify clusters in the data, using comorbidity information and demographic factors, led to solutions with many clusters that were difficult to interpret. Subsequently, the data were stratified by region of residence (metropolitan vs regional/rural) and separate two-step cluster analyses performed for Melbourne metropolitan residents and Victorian regional/rural residents. Area of residence was based on the Victorian Department of Health regions based on the local government area region of residence. ${ }^{19}$ This resulted in more meaningful clusters. The results of the cluster analyses are represented by a decision tree to show how admissions could be classified into a particular cluster.

The LOS (number of days for the first episode of care) for each episode was calculated and then compared across clusters using competing risks regression (Stata, V.11.2) ${ }^{20}$ Discharge to private residence was defined as the outcome of interest, while competing risks were defined as death, left against medical advice and separation to an aged care residential facility. Other separation types (eg, transition care, restorative care, statistical separations) were considered censored observations. The association between cluster membership and LOS was estimated using the subhazard ratio (SHR) with the cluster with the longest median LOS used for the reference category. The SHR is interpreted similarly to an $\mathrm{HR}$, in that an SHR $>1$ indicates an increased hazard of discharge to private residence, which corresponds to a shorter LOS.

\section{RESULTS}

There were 45374 episodes of admissions for falls in community-dwelling older adults that met the selection criteria. Table 1 presents the characteristics of these patients. The majority of admissions were for patients residing in metropolitan Melbourne (72.8\%). The distribution of episodes across metropolitan Melbourne and regional/rural Victoria did not change over the study period $\left(\chi^{2}(2)=3.66, p=0.16\right)$. More than two-thirds of the 
admissions were for women, and more than three-quarters were in people aged $75+$ years. Just under half of the admissions were for patients who were married or in a de facto relationship. Almost three-quarters had no comorbidities reported. The most commonly reported comorbidities in all cases included in this study were hypertensive disease (9.7\%), diabetes (6.9\%), dementia (4.7\%), renal disease $(4.1 \%)$ and cardiac dysrhythmias $(3.7 \%)$. These characteristics were similar in people residing in the metropolitan Melbourne area and those residing in regional/rural Victoria. In contrast, a higher proportion of admissions of Melbourne metropolitan residents were of patients born in a country other than Australia (40.1\%) compared with those residents in regional/rural Victoria (18.1\%). For these reasons, the remaining results are presented separately for metropolitan and regional/rural residents.

\section{Metropolitan clusters}

Admissions in the Melbourne metropolitan region clustered into five distinct groups (average silhouette $=0.3$ ). Sex, age group, marital status and comorbidities contributed strongly to membership, whereas country of birth was of less importance. Figure 1 demonstrates how Melbourne metropolitan resident admissions were classified into the five clusters using a decision tree. Group 1, representing $26.0 \%$ of admissions, comprised patients with comorbidities. Group 2 (21.8\% of admissions) were men without comorbidities. Groups 3 and $4(16.3 \%$ and $18.0 \%$ of admissions, respectively) were made up of women (married/de facto and single, respectively), aged between 65 and 84 years, without comorbidities. Group 5 represented $17.9 \%$ of admissions and comprised women aged $85+$ years without comorbidities.

\section{Regional/rural population clusters}

The analysis for admissions in regional/rural Victoria resulted in seven clusters (average silhouette $=0.5$ ), with all factors in table 1

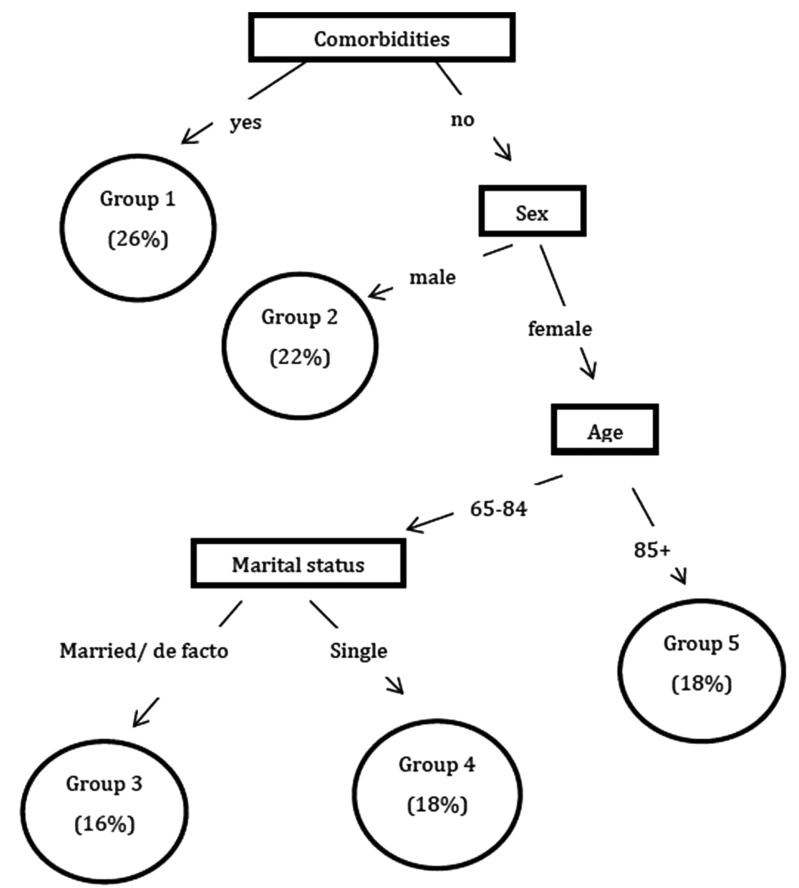

Figure 1 Classification of Melbourne metropolitan resident admissions into clusters. Group 1: all with comorbidities; group 2: men with no comorbidities; group 3: married 65-to-84-year-old women, no comorbidities; group 4: single 65-to-84-year-old women, no comorbidities; group 5: 85+-year-old women, no comorbidities. important for determining cluster membership. Figure 2 is a decision tree demonstrating how regional/rural Victorian residents can be classified into these seven clusters. Group 1 (18.3\% of admissions) comprised patients born outside of Australia. Groups 2 and $3(9.1 \%$ and $12.1 \%$ of admissions, respectively) were Australian-born patients with comorbidities; group 2 were those currently married or in a de facto relationship, while group 3 were not currently in a relationship. Group 4 represented $17.4 \%$ of admissions and comprised Australian-born men without comorbidities. Groups 5, 6 and 7 (15.0\%, 12.1\% and 16.1\% of admissions, respectively) were Australian-born women without comorbidities that differed according to marital status and age: group 5 members were single and aged 65-84 years, group 6 members were single and aged $85+$ years, while group 7 members were currently married or in a de facto relationship, irrespective of age.

\section{Length of stay}

The association between group membership and LOS was estimated using competing risks survival analysis. For admissions of patients residing in the Melbourne metropolitan area, all clusters were significantly different from each other $(p<0.001)$ in terms of LOS (table 2). Patients with comorbidities had the longest LOS, followed by (in order of descending LOS) women aged $85+$ years without comorbidities, single 65-to-84-year-old women without comorbidities, men without comorbidities and married 65-to-84-year-old women without comorbidities.

Comparisons of LOS across the different clusters of admissions of patients residing in regional/rural areas showed that all

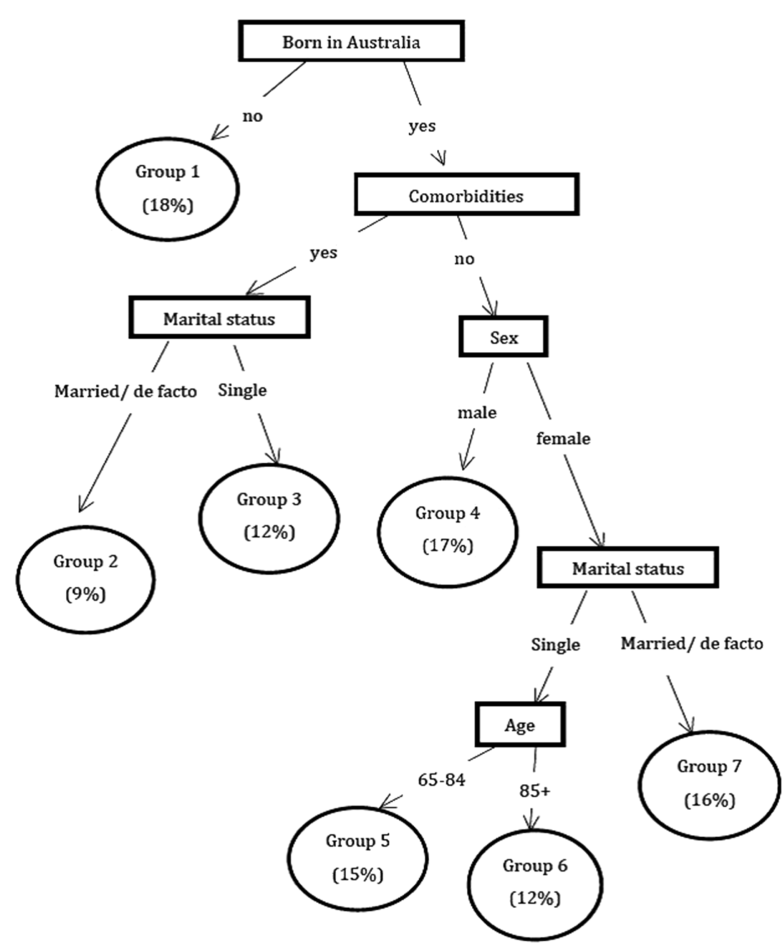

Figure 2 Classification of regional/rural Victorian resident admissions into clusters. Group 1: all overseas born; group 2: married Australian born, with comorbidities; group 3: single Australian born, with comorbidities; group 4: men, Australian born, no comorbidities; group 5: single 65-to-84-year-old women, Australian born, no comorbidities; group 6: single 85+-year-old women, Australian born, no comorbidities; group 7: married women, Australian born, no comorbidities. 
groups were significantly different from each other $(p<0.05)$ for all but one comparison. The comparison between Australian-born men without comorbidities (group 4) and single 65-to-84-year-old Australian-born women without comorbidities (group 5) did not differ significantly $(\mathrm{p}=0.08)$.

\section{DISCUSSION}

Several studies have highlighted the significant and increasing burden of hospitalised falls in older people. ${ }^{5}$ While previous epidemiological studies have demonstrated the need for policy and practice responses to falls prevention and identified individual falls risk factors, specific groups (other than age and gender) of older community-dwelling people who should be the target for falls interventions have not been identified. This study applied cluster analysis to identify groups of hospitalised fallers, based on demographic characteristics and the presence of comorbidities, who consume a disproportionate share of hospital resources and hence could be the focus of targeting of intervention programmes.

The cluster analysis identified different population subgroups of older people hospitalised for falls residing in the Melbourne metropolitan area compared with those for people residing in regional/rural Victoria. Most notably, country of birth was more important for predicting cluster membership for regional/rural admissions (18\% of regional/rural admissions were for patients born overseas) than metropolitan admissions (40\% of metropolitan admissions were for those born overseas). Reasons for these regional differences are unknown, but could include differential access to healthcare or provision of community services, different fall-injury risks in the two regions such as could be associated with the built environment or different population distribution of the factors in the model between metropolitan and regional areas.

Over a quarter of falls-related admissions were for people with comorbidities. Comorbidities have previously been shown to contribute independently to the risk of falling, sustaining a fall-related injury ${ }^{21}$ and to be prevalent in patients hospitalised for a fall or fall-related fracture (up to $37 \%$ ). ${ }^{22} 23$ The relatively high prevalence of comorbidities in patients hospitalised following a fall highlights the potential impact of these conditions on fall outcomes and opportunity to target falls prevention. Fallers with multiple comorbid chronic diseases are likely to have complex medical needs that are poorly understood. ${ }^{24}$ Focusing on people with specific comorbidity profiles may be an effective approach; however, further research is needed to determine whether proven falls prevention strategies need to be different for people with different numbers or types of comorbidity.

The first episode of hospital care LOS for people with comorbidities was significantly longer than that among clusters with no comorbidity. This finding is consistent with a recent study of community-dwelling older people aged $\geq 65$ years, hospitalised for a fall-related injury, in which the presence of comorbidities was significantly associated with increased LOS. ${ }^{24}$ Several studies of older people in the community have shown that comorbidities are strong determinants of mortality, ${ }^{25-27}$ but there has been limited research investigating the relationship between comorbid conditions and falls injury risk or outcomes. Our study suggests that one key target group for falls prevention should be older people with comorbidities, assuming that intervention strategies are just as effective for them as for other groups. It is also possible that targeting evidence-based falls interventions specifically to older people with comorbidities could reduce the impact this group has on fall-related hospital resources. Further work investigating the cost-effectiveness of falls prevention interventions and the need for complex interventions or integration of falls prevention into existing programmes for chronic disease ${ }^{28}$ for this subgroup would be beneficial.

In this study, clusters were also defined by individual demographic characteristics, such as place of birth or age. Women aged $85+$ years without comorbidities had longer LOS following a fall-related hospital admission compared with other subgroups without comorbidities (men and younger women) in metropolitan areas. In rural/regional areas, single women aged $85+$ years without comorbidities were also shown to have longer LOS following a fall-related hospital admission compared with other subgroups without comorbidities (men, younger single women and married women). This subgroup may have a greater risk of more severe injury or may be more likely to be living alone and therefore may need to stay in hospital longer before they can return home. Further analysis is needed to differentiate between the combined effects of gender, age, marital status and comorbidity on LOS. The ageing of the population ${ }^{13}$ and greater numbers of older women may have a significant impact on fall-related healthcare use and associated costs.

There are several limitations to this study. First, the presence of comorbidity was only classified as either present or absent. It is possible that the number, type and seriousness of the comorbidities may be more influential on cluster membership and LOS than whether or not comorbidity is present. However, cluster analyses become complex and difficult to interpret if too many factors are included. A study examining the prevalence and ramifications of specific combinations of chronic conditions suggested that specific comorbidity combinations can have large impacts on health or costs of care. ${ }^{24}$ Previous studies using the CCI, which takes into account the number and health burden of comorbid diseases, have shown a significant association between increasing CCI and in-hospital mortality. ${ }^{14} 29$ Interactions between existing comorbidities could compound health status and further impact on LOS, and distinct cluster patterns of comorbidity, such as stroke or cancer, have been previously identified in patients with fall-related injury. ${ }^{22}$ The current study provides additional evidence that the presence of comorbidities is important in terms of falls-related hospital admission frequency and LOS and warrant further investigation. Further work is needed to identify whether specific types or combinations of comorbidities account for disproportionate hospital resources with respect to fall-related injury. Understanding distinct cluster patterns of comorbidities would enable the effective targeting of falls prevention interventions.

Second, the prevalence of comorbid conditions could be underestimated in the VAED data. As the accuracy of the VAED capture of comorbidity has not been previously evaluated, it is not possible to estimate how this might affect the identified clusters and population profiles. However, recent research has used this same database to explore links between comorbidities and LOS. ${ }^{30}$ In addition, Australian coding standards for hospitalisation data require that only health conditions related to the current admission are recorded. While the low prevalence of comorbidity in this study was similar to a recent Victorian study, ${ }^{30}$ higher rates of comorbidities in older community-dwelling people (up to 50\%) have been reported in the USA and Europe. ${ }^{11} 31$

Third, while the VAED is subject to regular audits that indicate good-to-excellent diagnosis and procedure coding quality, ${ }^{32}$ it lacks a system-wide unique patient identifier. This means that we could only analyse unlinked episodes of care. To minimise the likelihood of including repeat admissions in our data set, we 
Table 2 Length of stay comparison across area and cluster membership

\begin{tabular}{|c|c|c|c|c|c|}
\hline Group & Description & $\begin{array}{l}\text { Median time to discharge to } \\
\text { private residence (days) }\end{array}$ & $\begin{array}{l}\text { Incidence rate } \\
\text { (discharges to private } \\
\text { residence per day) }\end{array}$ & $\begin{array}{l}\text { Subhazard } \\
\text { ratio }(95 \% \mathrm{Cl})\end{array}$ & p Value \\
\hline \multicolumn{6}{|c|}{ Melbourne metropolitan area } \\
\hline 1 & All with comorbidities & $25(9-80)$ & 0.030 & Reference & \\
\hline 2 & Men with no comorbidities & $3(1-14)$ & 0.146 & 3.15 (3.02 to 3.30$)$ & 0.000 \\
\hline 3 & Married 65-to-84-year-old women, no comorbidities & $3(1-11)$ & 0.166 & 3.51 (3.35 to 3.67$)$ & 0.000 \\
\hline 4 & Single 65-to-84-year-old women, no comorbidities & $4(1-18)$ & 0.128 & 2.84 (2.71 to 2.98$)$ & 0.000 \\
\hline 5 & 85+-year-old women, no comorbidities & $12(2-36)$ & 0.073 & 1.81 (1.72 to 1.91$)$ & 0.000 \\
\hline \multicolumn{6}{|c|}{ Regional/rural areas } \\
\hline 1 & All overseas born & $7(1-30)$ & 0.085 & 2.17 (1.97 to 2.38$)$ & 0.000 \\
\hline 2 & Married, Australian born with comorbidities & $17(7-59)$ & 0.039 & $1.16(1.03$ to 1.30$)$ & 0.011 \\
\hline 3 & Single, Australian born with comorbidities & $21(8-53)$ & 0.034 & Reference & \\
\hline 4 & Men, Australian born, no comorbidities & $4(1-13)$ & 0.124 & 2.83 (2.58 to 3.11$)$ & 0.000 \\
\hline 5 & Single 65-to-84-year-old women, Australian born, no comorbidities & $5(1-16)$ & 0.113 & 2.65 (2.41 to 2.92$)$ & 0.000 \\
\hline 6 & Single 85+-year-old women, Australian born, no comorbidities & $11(3-29)$ & 0.067 & $1.63(1.46$ to 1.81$)$ & 0.000 \\
\hline 7 & Married women, Australian born, no comorbidities & $4(1-13)$ & 0.135 & 3.06 (2.78 to 3.36$)$ & 0.000 \\
\hline
\end{tabular}

applied case selection restriction criteria that have previously been shown to be optimal for identifying incident falls cases. ${ }^{33}$ Internally linked (person-identifying) hospital discharge data on hip fractures provide a more accurate estimate of hip fracture incidence and the prevalence of some comorbid conditions than episode-based data. ${ }^{22}$ The lack of linked hospitalisation data did not allow us to use retrospective data to improve the identification of comorbid conditions. ${ }^{34}$

Finally, cluster cohesion was higher in regional/rural Victoria compared with the Melbourne metropolitan region; for regional/rural clusters, the silhouette measure indicated the solution was good, while for metropolitan clusters, the solution was only fair. The inclusion of different demographic characteristics and fall risk factors may have resulted in more cohesive clusters and more accurate reflection of the impact of comorbidity and other characteristics on fall outcomes. However, we were restricted by the data available in the VAED. Further research is needed to understand the association of comorbidity with increased healthcare use and would be enhanced by using linked data and comorbidity measures such as the CCI.

Importantly, this analysis has identified a number of different groups of older people with similar characteristics at whom prevention programmes could be targeted. In regional/rural areas, targeting people born overseas may be a feasible falls prevention strategy and could be more effective than a generalised falls prevention programme aimed at all community-dwelling people. Cost-benefit analyses and further epidemiological modelling should be undertaken to determine whether greater falls reductions in these specific subgroups are needed in order to achieve the same reduction in hospitalisations that might be achieved with a more multifaceted population-based approach. It is also currently unknown whether falls interventions, shown to be effective in general patient randomised controlled trials, will result in the same level of falls reduction among the specific subgroups identified in this study, such as those with comorbidity.

\section{CONCLUSION}

This study has identified distinct clusters of people, based on selected individual and demographic characteristics, who are admitted to hospital for falls and who use the greatest proportion of hospital resources. Patients with comorbidity, and women aged 85 years and older without comorbidities, stay in hospital longer following a fall than do other population clusters and hence consume a disproportionate share of hospital resources for fall-related hospital admissions. For this reason, subgroup targeting at the population level could begin with these groups. Overall, these findings have important implications for the targeting and implementation of falls prevention interventions for community-dwelling older people.

\section{What is already known on the subject}

- Previous epidemiological studies have demonstrated the need for policy and practice responses to falls prevention and identified individual falls risk factors.

- Specific groups (other than age and gender) of older community-dwelling people who should be the target for falls interventions have not been identified.

- It is recognised that older people with comorbidities have worse health outcomes following an injurious fall than do those without comorbidities.

\section{What this study adds}

- Different population subgroups of older people hospitalised for falls prevention were identified in residents of metropolitan areas and regional/rural areas.

- Patients with comorbidity, and older women, stay the longest in hospital following a fall and hence consume a disproportionate share of hospital resources.

- Targeting these subgroups, using a population-level approach to falls prevention, could have a large impact on health outcomes and hospital resource use.

Acknowledgements Angela Clapperton and Jane Hayman from the Monash Injury Research Institute, Monash University, assisted with data definitions, data extraction and initial data analyses. ACRISP is one of only four research centres 
internationally to be recognised and supported by the International Olympic Committee (IOC) as an IOC Research Centre for the Prevention of Injury and Promotion of Athlete Health.

Twitter Follow Caroline Finch at @CarolineFinch and Anna Wong Shee at @annawongshee

Contributors CFF, LD, KH, TPH and LC conceived the study, contributed to the analysis plan and to the paper writing. AWS had major responsibility for the writing of the introduction and discussion sections. KS undertook the data analysis, developed the analysis plan and contributed to the writing of the paper, especially the methods and results sections.

Funding This study was funded by a partnership project grant (ID 546282) from the Australian Government National Health and Medical Research Council (NHMRC), with additional funding from the Victorian Department of Health. CFF was supported by NHMRC Principal Research Fellowships (ID 565900 and ID 1058737). AWS was supported by an Emeritus Professor Robert HT Smith Postdoctoral Research Fellowship from Federation University Australia.

Competing interests None.

Ethics approval Victorian Department of Health HREC.

Provenance and peer review Not commissioned; externally peer reviewed.

Open Access This is an Open Access article distributed in accordance with the Creative Commons Attribution Non Commercial (CC BY-NC 4.0) license, which permits others to distribute, remix, adapt, build upon this work non-commercially, and license their derivative works on different terms, provided the original work is properly cited and the use is non-commercial. See: http://creativecommons.org/ licenses/by-nc/4.0/

\section{REFERENCES}

1 Gillespie LD, Robertson MC, Gillespie WJ, et al. Interventions for preventing falls in older people living in the community. Cochrane Database Syst Rev 2012(9): CD007146.

2 Campbell A, Borrie M, Spears GF, et al. Circumstances and consequences of falls experienced by a community population 70 years and over during a prospective study. Age Ageing 1990;19:136-41.

3 Hindmarsh DM, Hayen A, Finch CF, et al. Relative survival after hospitalisation for hip fracture in older people in New South Wales, Australia. Osteoporos Int 2009;20:221-9.

4 Cumming RG, Salkeld G, Thomas $M$, et al. Prospective study of the impact of fear of falling on activities of daily living, SF-36 scores, and nursing home admission. J Gerontol A Biol Sci Med Sci 2000;55:M299-305.

5 Mitchell R, Close J, Cameron I, et al. Fall-related sub-acute and non-acute care and hospitalised rehabilitation episodes of care: What is the injury burden? Aust Health Rev 2013:37:348-55.

6 Moller J. Projected costs of fall related injury to older persons due to demographic change in Australia. Report to the Commonwealth Department of Health and Ageing under the National Falls Prevention for Older People Initiative. Canberra. https://www. health.gov.au/internet/main/publishing.nsf/650f3eec0dfb990fca25692100069854/ d2f2014ed7944d70ca257bf000199e5d/\$FILE/falls_costs.pdf (accessed 19 Sep 2013).

7 Bradley C. Hospitalisations due to falls by older people, Australia 2008-09. Injury Research and Statistics Series Number 62. Canberra: Australian Institute of Health and Welfare, 2012. http://www.aihw.gov.au/publication-detail/?id=10737421923 (accessed 9 Sep 2013).

8 Haines T, Hill AM, Hill K, et al. Patient education to prevent falls among older hospital inpatients: A randomized controlled trial. Arch Intern Med 2011;171:516-24.

9 Noonan RK, Sleet DA, Stevens JA. Closing the Gap: a research agenda to accelerate the adoption and effective use of proven older adult fall prevention strategies. J Safety Res 2011:42:427-30.

10 Tetroe JM, Graham ID, Scott V. What does it mean to transform knowledge into action in falls prevention research? Perspectives from the Canadian Institutes of Health Research. J Safety Res 2011;42:423-6.
11 Mathers C, Murray C, Ezzati M, et al. Population health metrics: crucial inputs to the development of evidence for health policy. Popul Health Metr 2003;1:6-9.

12 Day L, Finch CF, Hill K, et al. A protocol for evidence-based targeting and evaluation of statewide strategies for preventing falls among community-dwelling older people in Victoria, Australia. Inj Prev 2011;17:e3.

13 Australian Bureau of Statistics. 3235.0-Population by age and sex, regions of Australia, 2011. Canberra: Australian Bureau of Statistics, 2012. http://www.abs.gov. au/ausstats/abs@.nsf/Products/3235.0 2011 Main+Features Victoria\#PARALINK6 (accessed 30 Oct 2013).

14 Sundararajan V, Henderson T, Perry C, et al. New ICD-10 version of the Charlson comorbidity index predicted in-hospital mortality. J Clin Epidemio/ 2004;57:1288-94.

15 Zhang T, Ramakrishnan R, Livny M. BIRCH: A new data clustering algorithm and its applications. Data Min Knowl Discov 1997;1:141-82.

16 Chiu T, Fang D, Chen J, et al. A robust and scalable clustering algorithm for mixed type attributes in large database environment. Proceedings of the seventh Association for Computing Machinery (ACM) International Conference on Knowledge Discovery and Data Mining, San Francisco, California. ACM; 2001. p. 263-8. http://www.kdd.org/ (accessed 25 Sep 2013).

17 Rousseeuw PJ. Silhouettes: a graphical aid to the interpretation and validation of cluster analysis. J Comput Appl Math 1987;20:53-65.

18 Mooi E, Sarstedt M. A concise guide to market research: the process, data and methods using IBM SPSS statistics. Int J Mark Res 2011;53:563-4.

19 Department of Health. Our regions: State Government of Victoria; 2014. http:// www.health.vic.gov.au/regions/ (accessed 15 Feb 2014).

20 Cleves M, Gould W, Gutierrez RG, et al. An introduction to survival analysis using Stata. 3rd edn. USA: Stata Press, 2010.

21 Tinetti $M$, Kumar $C$. The patient who falls: "It's always a trade-off". JAMA 2010;303:258-66.

22 Vu T, Day L, Finch CF. Linked versus unlinked hospital discharge data on hip fractures for estimating incidence and comorbidity profiles. BMC Med Res Methodol 2012;12:113-20

23 Vu T, Finch CF, Day L. Patterns of comorbidity in community-dwelling older people hospitalised for fall-related injury: a cluster analysis. BMC Geriatr 2011;11:45-54

24 Vogeli C, Shields A, Lee T, et al. Multiple chronic conditions: prevalence, health consequences, and implications for quality, care management, and costs. J Gen Intern Med 2007;22:391-5.

25 Camilloni L, Farchi S, Rossi PG, et al. Mortality in elderly injured patients: the role of comorbidities. Int J Inj Contr Saf Promot 2008;15:25-31.

26 Masud T, Binkley N, Boonen S, et al. Official positions for FRAX® clinical regarding falls and frailty: Can falls and frailty be used in FRAX®?. J Clin Densitom 2011;14:194-204.

27 Vestergaard P, Rejnmark L, Mosekilde L. Increased mortality in patients with a hip fracture-effect of pre-morbid conditions and post-fracture complications. Osteoporos Int 2007; 18:1583-93.

28 Department of Human Services. HARP Hospital Admissions Risk Program Victoria: Department of Health; 2013. http://www.health.vic.gov.au/harp/about.htm (accessed 1 Oct 2013).

29 Hindmarsh D, Loh M, Finch CF, et al. Effect of comorbidity on relative survival following hospitalisation for fall-related hip fracture in older people. Aust J Ageing 2014;33:E1-7.

30 Vu T, Day L, Finch CF. The burden of hospitalised fall-related injury in community-dwelling older people in Victoria: a database study. Aust NZ J Public Health 2014;38:128-33.

31 Lang P, Heitz D, Hédelin G, et al. Early markers of prolonged hospital stays in older people: a prospective, multicenter study of 908 Inpatients in French acute hospitals. J Amer Geriatr Soc 2006;54:1031-9.

32 Henderson T, Shepheard J, Sundararajan V. Quality of diagnosis and procedure coding in ICD-10 adminstrative data. Med Care 2006:44:1011-9.

33 Boufous $\mathrm{S}$, Finch $\mathrm{C}$, Close J, et al. Hospital admissions following presentations to emergency departments for a fracture in older people. Inj Prev 2007;13:211-4.

34 Preen DB, Holman CDJ, Spilsbury K, et al. Length of comorbidity lookback period affected regression model performance of administrative health data. Journal of Clinical Epidemiology 2006;59:940-6. 